\title{
The Signature of Low Redshift Large-Scale Structure in the Cosmic Microwave Background
}

\author{
David Spergel* \\ Department of Astrophysical Sciences, Princeton University \\ E-mail: dns@astro.princeton.edu
}

\begin{abstract}
As microwave background photons propagate from the surface of last scatter to our telescopes, they are affected by four distinct processes in the low redshift universe: gravitational lensing, the thermal Sunyaev-Zeldovich (tSZ) effect, the kinematic Sunyaev Zeldovich (kSZ) effect and the intervening Sachs Wolfe effect (ISW). This talk will focus on the kSZ and ISW effect. I will discuss the cross-correlations between the large-scale distribution of galaxies and these two effects and show how current and future measurements can be used to probe the growth rate of structure and gravitational physics on large-scales.
\end{abstract}

Frontiers of Fundamental Physics 14 - FFP14,

15-18 July 2014

Aix Marseille University (AMU) Saint-Charles Campus, Marseille

${ }^{*}$ Speaker. 